\title{
Oxygen Ion Conductivity and Composition at the Grain Boundaries of Ca Doped $\mathrm{CeO}_{2}$
}

\author{
Brittney M. Hauke ${ }^{1}$, Barnaby D. A. Levin ${ }^{1}$, and Peter A. Crozier ${ }^{1}$ \\ 1. School for the Engineering of Matter, Transport and Energy, Arizona State University, Tempe, USA.
}

Polycrystalline electro-ceramics play an important role in solid oxide fuel cells (SOFCs) and there is considerable interest in improving the oxygen ion conduction for intermediate temperature applications. The oxygen ion conduction may be significantly degraded by the presence of grain boundaries; for example the grain boundary conductivity can be $10^{2}-10^{6}$ times lower than the grain conductivity in $\mathrm{CeO}_{2}$ based materials [1]. It is important to understand how the atomic structure and composition at these interfaces relates to the ionic conductivity. Here, a series of electrolytes of $\mathrm{CeO}_{2}$ doped with $\mathrm{Ca}^{+2}$ was investigated with atomic resolution imaging and spectroscopy to determine the correlations between grain boundary structure, composition, and ionic conductivity.

$\mathrm{CeO}_{2}$ powders of $2 \%, 5 \%$, and $10 \%$ molar concentrations of $\mathrm{Ca}$ were synthesized via spray drying, as described by Sharma et al. [2]. The recovered powder was calcined at $500{ }^{\circ} \mathrm{C}$ for two hours with a fivehour ramp to temperature. It was then pressed into pellets by compacting $1 \mathrm{~g}-1.5 \mathrm{~g}$ of powder in a hardened steel die at 5000 psi for one minute under uniaxial compression. The pellets were sintered in an alumina crucible at $1400^{\circ} \mathrm{C}$ for 24 hours with a $2.5^{\circ} \mathrm{C} / \mathrm{min}$ heating rate. Specimens were prepared for STEM by mechanical dimpling followed by precision ion polishing. Electron microscopy was done with a JEOL ARM200F aberration-corrected STEM. AC impedance spectroscopy (ACIS) was also done on the pellets via a Gamry Reference 3000 potentiostat while inside a Lindberg Blue tube furnace heated from $50^{\circ} \mathrm{C}$ to $700{ }^{\circ} \mathrm{C}$. Impedance spectra were acquired at each dwell time, which were then fitted via an equivalent circuit model. The corresponding resistance values were used to calculate the activation energy of both the grain and the grain boundary.

A typical Nyquist plot is shown in Figure 1, which plots the real and imaginary components of the impedance of a $10 \%$ (i.e. $\mathrm{Ca}_{0.1} \mathrm{Ce}_{0.9} \mathrm{O}_{2-\square}$ ) pellet at $400^{\circ} \mathrm{C}$ using a R-2RQ equivalent circuit fit. Figure 2a shows a low magnification STEM ADF image of the $10 \%$ pellet where multiple grain boundaries can be seen at the edge of the specimen. Figure $\mathbf{2 b}$ is a higher magnification STEM image of one particular triple point. Figure 3a shows two electron energy-loss (EELS) spectra, one taken at the grain interior and one at a single grain boundary. The point scan at the grain boundary shows a substantial increase in the calcium $\mathrm{L}_{23}$ edge. Figure 3b shows an EELS map taken at the triple point from Figure 2b, where Ce is red and $\mathrm{Ca}$ is green. Similar measurements will be performed on the $2 \%$ and $5 \%$ samples and the grain boundary atomic and elemental structure will be correlated with the ionic conductivity [3].

\section{References:}

[1] Bowman, W. et al, Nanoscale 9 (2017), p. 17293.

[2] Sharma, V. et al, Chemical Physics Letters 495 (2010), p. 280.

[3] We gratefully acknowledge support of NSF grant WZS0118 and Arizona State University's John M. Cowley Center for High Resolution Electron Microscopy. 


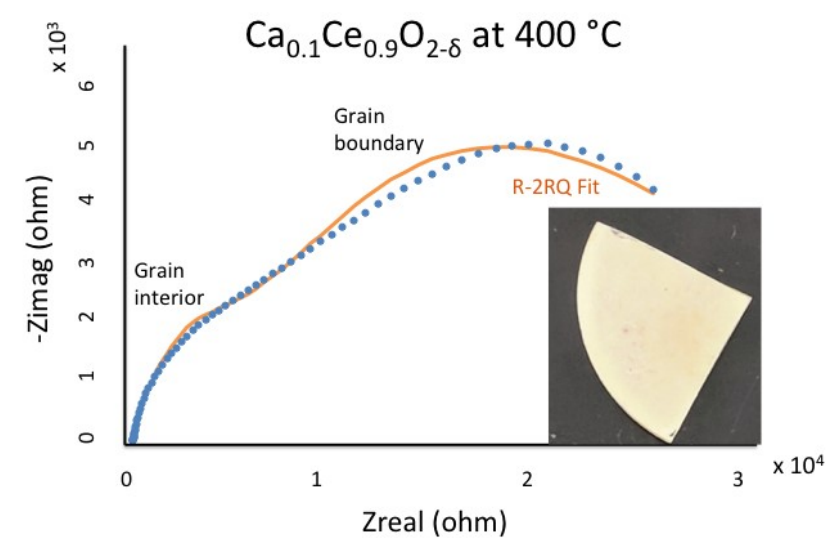

Figure 1. Sample Nyquist plot taken at $400^{\circ} \mathrm{C}$ fitted using a R-2RQ equivalent circuit model and image of one quarter of a pellet after ACIS.

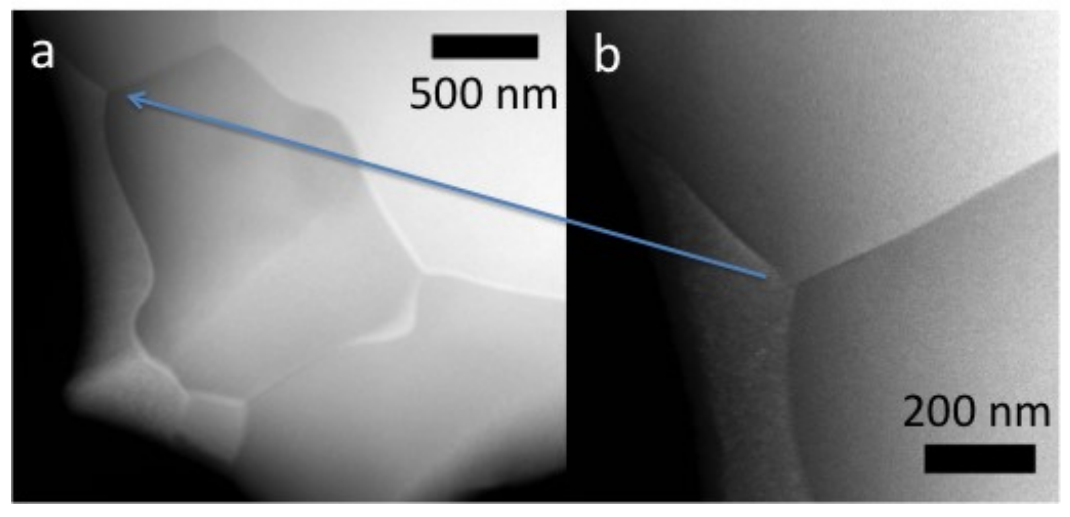

Figure 2a. Low magnification STEM image of $10 \% \mathrm{CaCeO}_{2}$ specimen. 2b. Higher magnification STEM image showing a triple point.

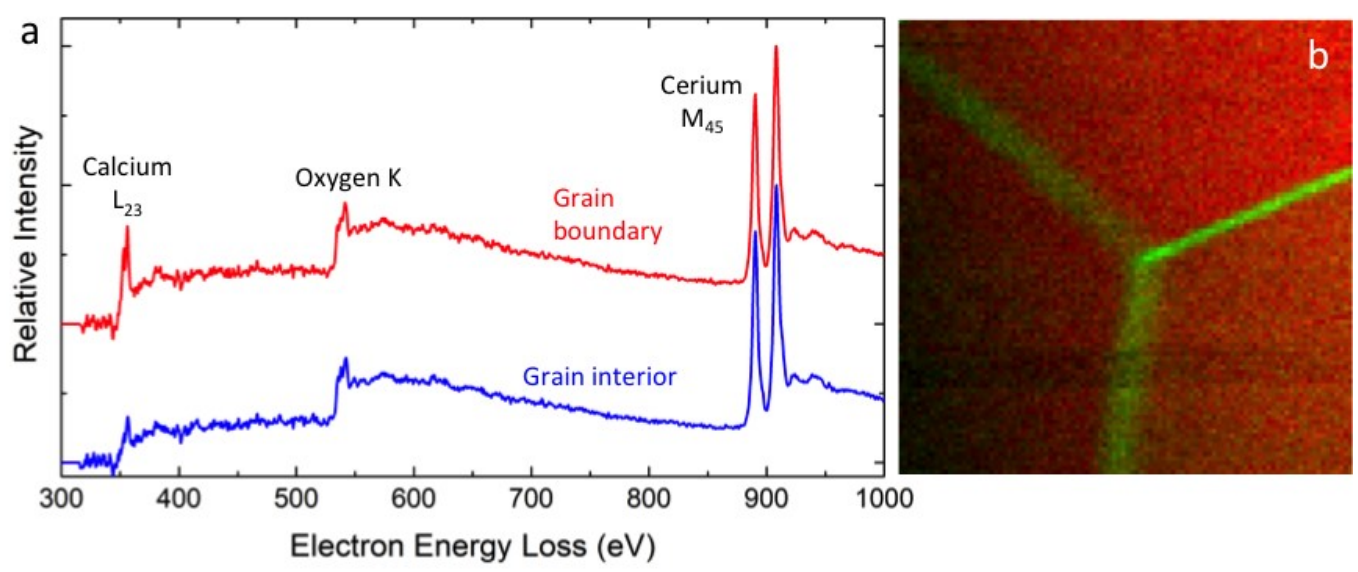

Figure 3a. EELS spectra from the grain boundary and grain interior. 3b. EELS elemental map of Ca (green) and Ce (red) at the center of the triple point shown in $\mathbf{2} \mathbf{b}$. 